\title{
AD HOC CONSULTANTS - 2021
}

Adolfo Jesiel Siebra - Universidade Federal do Ceará, Fortaleza-CE, Brasil

Adriana Garcia Gonçalves - Universidade Federal de São Carlos, São Carlos-SP, Brasil

Adriana Martins Saur - Universidade de São Paulo, Ribeirão Preto-SP, Brasil

Alessandra Brunoro Motta Loss - Universidade Federal do Espírito Santo, Vitória-ES, Brasil

Alessandra da Silva Souza de Carvalho - Universidade do Porto, Porto, Portugal

Alline Alves de Sousa - Universidade Federal do Espírito Santo, Vitória-ES, Brasil

Ana Cristina Vidigal Soeiro - Universidade Federal do Pará, Belém-PA, Brasil

Ana Isabel Martins Sani - Universidade Fernando Pessoa, Porto, Portugal

Ana Luiza Gomes Pinto Navas - Faculdade de Ciências Médicas da Santa Casa de São Paulo, São Paulo-SP, Brasil

André Luiz Damião de Paula - Universidade de São Paulo, Ribeirão Preto-SP, Brasil

André Luiz Monezi Andrade - Pontifícia Universidade Católica de Campinas, Campinas-SP, Brasil

Andrea Ruzzi Pereira - Universidade Federal do Triângulo Mineiro, Uberaba-MG, Brasil

Andreia Schmidt - Universidade de São Paulo, Ribeirão Preto-SP, Brasil

Angela Helena Marin - Universidade Federal do Rio Grande do Sul, Porto Alegre-RS, Brasil

Angelina Lettiere Viana - Universidade de São Paulo, Ribeirão Preto-SP, Brasil

Anna Paula Uziel - Universidade do Estado do Rio de Janeiro, Rio de Janeiro-RJ, Brasil

Antonio Abel Pires - Universidade do Porto, Porto, Portugal

Betânia Alves Veiga Dell'Agli - Centro Universitário das Faculdades Associadas de Ensino, Santo André-SP, Brasil

Carla Guanaes-Lorenzi - Universidade de São Paulo, Ribeirão Preto-SP, Brasil

Caroline Francisca Eltink - Universidade Paulista, Ribeirão Preto-SP, Brasil

Celia Regina Rangel Nascimento - Universidade Federal do Espírito Santo, Vitória-ES, Brasil

Clarice Medeiros - Universidade Veiga de Almeida, Rio de Janeiro-RJ, Brasil

Clarissa Mendonça Corradi-Webster - Universidade de São Paulo, Ribeirão Preto-SP, Brasil

Clarissa Tochetto de Oliveira - Universidade Federal do Rio Grande do Sul, Porto Alegre-RS, Brasil

Claudia Benedita dos Santos - Universidade de São Paulo, Ribeirão Preto-SP, Brasil

Claudia Maria Simões Martinez - Universidade Federal de São Carlos, São Carlos-SP, Brasil

Cristina Maria D’Antona Bachert - Universidade de Sorocaba, Sorocaba-SP, Brasil

Cynthia Cassoni - Universidade do Estado do Rio de Janeiro, Rio de Janeiro-RJ, Brasil 
Daniel Bartholomeu - Centro Universitário FIEO, Osasco-SP, Brasil

Daniele Carolina Lopes - Universidade de São Paulo, São Carlos-SP, Brasil

Davi do Socorro Barros Brasil - Universidade Federal do Pará, Belém-PA, Brasil

Debora de Hollanda Souza - Universidade Federal de São Carlos, São Carlos-SP, Brasil

Denise Falcke - Universidade do Vale do Rio dos Sinos, São Leopoldo-RS, Brasil

Denise Medeiros Ribeiro Salles - Universidade Federal Fluminense, Niterói-RJ, Brasil

Denize Rosana Rubano - Pontifícia Universidade Católica de São Paulo, São Paulo-SP, Brasil

Diene Monique Carlos - Universidade Federal de São Carlos, São Carlos-SP, Brasil

Eduardo Name Risk - Universidade Federal de São Carlos, São Carlos-SP, Brasil

Elizabeth Joan Barham - Universidade Federal de São Carlos, São Carlos-SP, Brasil

Emanuela Bezerra Torres Mattos - Universidade Federal de São Paulo, Santos-SP, Brasil

Érika Arantes de Oliveira-Cardoso- Universidade de São Paulo, Ribeirão Preto-SP, Brasil

Evanisa Helena Maio de Brum - Centro Universitário CESMAC, Maceió-AL, Brasil

Fábio Scorsolini-Comin - Universidade de São Paulo, Ribeirão Preto-SP, Brasil

Fátima Cristina Regis Martins de Oliveira - Universidade do Estado do Rio de Janeiro, Rio de Janeiro-RJ, Brasil

Felipe Valentini - Universidade São Francisco, Campinas-SP, Brasil

Fernanda de Vargas - Pontifícia Universidade Católica do Rio Grande do Sul, Porto Alegre-RS, Brasil

Fernando Antônio de Barros Júnior - Universidade de São Paulo, Ribeirão Preto-SP, Brasil

Giana Bitencourt Frizzo - Universidade Federal do Rio Grande do Sul, Porto Alegre-RS, Brasil

Gisele Cristina Resende - Universidade Federal do Amazonas, Manaus-AM, Brasil

Guilherme Welter Wendt - Universidade Estadual do Oeste do Paraná, Francisco Beltrão-PR, Brasil

Heloísa Gonçalves Ferreira - Universidade do Estado do Rio de Janeiro, Rio de Janeiro-RJ, Brasil

Inês Maria Guimarães Nascimento - Universidade do Porto, Porto, Portugal

Ingrid Finger - Universidade Federal do Rio Grande do Sul, Porto Alegre-RS, Brasil

Irene Cadime - Universidade do Minho, Braga, Portugal

Isabela Maria Magali Mello Vieira - Universidade do Estado do Rio de Janeiro, Rio de Janeiro-RJ, Brasil

Jéssica Aline Rovaris - Universidade Estadual Paulista Júlio de Mesquita Filho, Bauru-SP, Brasil

Joanna Gonçalves de Andrade Tostes - Universidade Federal de Juiz de Fora, Juiz de Fora-MG, Brasil

João Carlos Caselli Messias - Pontifícia Universidade Católica de Campinas, Campinas-SP, Brasil 
João Cesar de Freitas Fonseca - Pontifícia Universidade Católica de Minas Gerais, Belo Horizonte-MG, Brasil

Jorge Fernando Pereira Sinval - Instituto Universitário de Lisboa, Lisboa, Portugal

José Aparecido da Silva - Universidade de São Paulo, Ribeirão Preto-SP, Brasil

José Augusto Evangelho Hernandez - Universidade do Estado do Rio de Janeiro, Rio de Janeiro-RJ, Brasil

José Egídio Oliveira - Escola Superior de Saúde do Alcoitão, Alcabideche, Portugal

Jose Francisco Miguel Henriques Bairrão - Universidade de São Paulo, Ribeirão Preto-SP, Brasil

Julliane Messias Cordeiro Sampaio - Centro Universitário de Brasília, Brasília-DF, Brasil

Katia Paschoali Miguel - Universidade de Ribeirão Preto, Ribeirão Preto-SP, Brasil

Kelly Graziani Giacchero Vedana - Universidade de São Paulo, Ribeirão Preto-SP, Brasil

Laura Camargo Macruz Feuerwerker - Universidade de São Paulo, São Paulo-SP, Brasil

Léia Maria Erlich Ruwer - Faculdade IBGEN - Instituto Brasileiro de Gestão de Negócios- Porto Alegre, RS - Brasil

Leonardo Rodrigues Sampaio - Universidade Federal do Vale do São Francisco, Petrolina-PE, Brasil

Letícia Lovato Dellazzana-Zanon - Pontifícia Universidade Católica de Campinas, Campinas-SP, Brasil

Lígia Braun Schermann - Universidade Luterana do Brasil, Canoas-RS, Brasil

Liliana Filipa Vale Costa - Universidade de Aveiro, Aveiro, Portugal

Luciana Carla dos Santos Elias - Universidade de São Paulo, Ribeirão Preto-SP

Luciana Mendonça Alves - Universidade de Minas Gerais, Belo Horizonte-MG, Brasil

Luciana Mourão Cerqueira e Silva - Universidade Salgado de Oliveira, Niterói-RJ

Lucienia Libania Pinheiro Martins - Universidade Federal do Piauí, Teresina-PI, Brasil

Ludmyla de Souza Alves - Universidade Federal de São Carlos, São Carlos-SP, Brasil

Lupicínio Íñiguez Rueda - Universitat Autònoma de Barcelona, Barcelona, Espanha.

Maicon Rodrigues Albuquerque - Universidade Federal de Minas Gerais, Belo Horizonte-MG, Brasil

Maíra Stivaleti Colombarolli - Universidade de São Paulo, Ribeirão Preto-SP, Brasil

Manoel Antônio dos Santos - Universidade de São Paulo, Ribeirão Preto-SP, Brasil

Marcelo Afonso Ribeiro - Universidade de São Paulo, São Paulo-SP, Brasil

Márcia Calixto dos Santos - Centro Universitário Salesiano de São Paulo, São Paulo-SP, Brasil

Márcia Maria Peruzzi Elia da Mota - Universidade do Estado do Rio de Janeiro, Rio de Janeiro-RJ, Brasil

Marciana Fernandes Moll - Universidade de Uberaba, Uberaba-MG, Brasil

Marcos Vinicius Brunhari - Universidade do Estado do Rio de Janeiro, Rio de Janeiro-RJ, Brasil 
Maria Cristina Triguero Veloz Teixeira - Universidade Presbiteriana Mackenzie, São Paulo-SP, Brasil Maria Isabel Patrício de Carvalho Pedrosa - Universidade Federal de Pernambuco, Recife-PE, Brasil Maria Manuela Martins - Escola Superior de Enfermagem do Porto, Porto, Portugal Maria Odília Teixeira - Universidade de Lisboa, Lisboa, Portugal Maria Paula Foss - Universidade de São Paulo, Ribeirão Preto-SP, Brasil Maria Regina Maluf - Pontifícia Universidade Católica de São Paulo, São Paulo-SP, Brasil Marilsa de Sá Rodrigues - Universidade de Taubaté, Taubaté-SP, Brasil Marina Bittencourt Bandeira - Universidade Federal de São João del Rei, São João del Rei-MG, Brasil Marina Simões Flório Ferreira Bertagnoli - Universidade de São Paulo, Ribeirão Preto-SP, Brasil Marina Kohlsdorf - Centro Universitário de Brasília, Brasília-DF, Brasil Marina Menezes - Universidade Federal de Santa Catarina, Florianópolis-SC, Brasil Marina Rezende Bazon - Universidade de São Paulo, Ribeirão Preto-SP, Brasil Marina Zanella Delatorre - Universidade Federal do Rio Grande do Sul, Porto Alegre-RS, Brasil Marlene de Cássia Trivellato-Ferreira - Centro Universitário Barão de Mauá, Ribeirão Preto-SP, Brasil Marlene Fagundes Carvalho Gonçalves - Universidade de São Paulo, Ribeirão Preto-SP, Brasil Marta Regina Gonçalves Correia Zanini - Centro Universitário das Faculdades Associadas de Ensino, São João da Boa Vista-SP, Brasil Mauricio Andres Lopez Cruz - Universidad de Chile, Santiago, Chile Mayra Antonelli-Ponti - Universidade de São Paulo, Ribeirão Preto-SP, Brasil Nadia Maria Ribeiro Salomão - Universidade Federal da Paraíba, João Pessoa-PB, Brasil Patricia Alvarenga - Universidade Federal da Bahia, Salvador-BA, Brasil Paulo Cesar Azevedo Dias - Universidade Católica Portuguesa, Braga, Portugal Paulo Henrique Ferreira Bertolucci - Universidade Federal de São Paulo, São Paulo-SP, Brasil Ricardo Burg Ceccim - Universidade Federal do Rio Grande do Sul, Porto Alegre-RS, Brasil Rosana Aparecida Spadoti Dantas - Universidade de São Paulo, Ribeirão Preto-SP, Brasil Rosane Pilot Pessa - Universidade de São Paulo, Ribeirão Preto-SP, Brasil Rosângela Andrade Aukar de Camargo - Universidade de São Paulo, Ribeirão Preto-SP, Brasil Shyrlleen Christieny Assunção Alves - Centro Universitário do Leste de Minas Gerais, Ipatinga-MG, Brasil Silvia Miranda Amorim - Universidade Federal de Minas Gerais, Belo Horizonte-MG, Brasil Sofia Santos - Universidade de Lisboa, Lisboa, Portugal 
Sônia Maria Guedes Gondim - Universidade Federal da Bahia, Salvador-BA, Brasil

Sonia Regina Loureiro - Universidade de São Paulo, Ribeirão Preto-SP, Brasil

Susana Maria Gonçalves Coimbra - Universidade do Porto, Porto, Portugal

Susana Nunez Rodriguez - Universidade Salgado de Oliveira, Niterói-RJ, Brasil

Sylvia Domingos Barrera - Universidade de São Paulo, Ribeirão Preto-SP, Brasil

Talita Pereira Dias - Centro Universitário de Votuporanga, Votuporanga-SP, Brasil

Tania Mara Marques Granato - Pontifícia Universidade Católica de Campinas, Campinas-SP, Brasil

Tássia Ghissoni Pedroso - Universidade de São Paulo, Ribeirão Preto-SP, Brasil

Tatiana de Cássia Nakano - Pontifícia Universidade Católica de Campinas, Campinas-SP, Brasil

Teresa Limpo - Universidade do Porto, Porto, Portugal

Thiago Ferreira Sousa - Universidade Federal do Recôncavo da Bahia, Amargosa-BA, Brasil

Thiago Medeiros Cavalcanti - Universidade Federal de Campina Grande, Cajazeiras-PB, Brasil

Tiago Alfredo da Silva Ferreira - Universidade Federal da Bahia, Salvador-BA, Brasil

Valéria Salek Ruiz - Petróleo Brasileiro - Rio de Janeiro - Matriz, Saúde Segurança e Meio Ambiente, Rio de Janeiro-RJ, Brasil Vanessa Barbosa Romera Leme - Universidade do Estado do Rio de Janeiro, Rio de Janeiro-RJ, Brasil

Walter Mariano de Faria Silva Neto - Universidade Federal do Triângulo Mineiro, Uberaba-MG, Brasil

Wanderlei Abadio de Oliveira - Pontifícia Universidade Católica de Campinas, Campinas-SP, Brasil 